NEWS AND VIEWS

\title{
Metro-scientists; taking sensitivity to new levels
}

\author{
Graeme Doran \\ Massachusetts Institute of Technology, USA
}

Microarrays have greatly increased the potential data that can be generated from a single experiment, and this lure has led to a great increase in the availability and application of such technology. As medical researchers recognize the diagnostic potential of such massively parallel high-throughput technologies, microarrays are increasingly being used to predict prognosis and response to specific therapies for a range of tumours in a clinical setting. The marketing success of such microfabrication may to some extent mask the inherent limitations associated with understanding and validating large amounts of array generated data. New developments in nanotechnologies and enhancements of existing techniques promise to improve the sensitivity and ability to simultaneously analyse a greater number of array generated targets in model systems. This level of verification is important in understanding the physiological relevance of microarray generated expression profiles.

\section{NUCLEIC ACID DRIVEN IMMUNO- AMPLIFICATION AND BARCODE DETECTION}

The basic principles for the detection of proteins via antibody-conjugated DNA probes have been long established (Sano et al, 1992), but technical limitations have prevented the widespread use of a methodology that offers a 100-10,000 fold enhancement over conventional enzyme linked immunoabsorbent assays (ELISAs). The primary obstacles in developing this technique have lay with synthesis of protein-DNA conjugates and eliminating high background signals due to DNA release or contamination. New methodological strategies based upon immuno-PCR, have capitalized upon the multiplexing potential afforded by different DNA sequences to offer highly parallel target analysis. Immuno-rolling circle amplification (RCA) extends an oligonucleotide primer covalently attached to the detection antibody to linearly amplify a circular DNA template. Although not reaching the same sensitivity of the exponentially amplified PCR technique, multiplex analysis of the expression profile of 11 cytokines has been demonstrated using fluorescent probes, and up to
75 cytokines from human dendritic cells were simultaneously analysed with femtomolar sensitivity via a microarray detection platform (Schweitzer et al, 2000; Mullenix et al, 2004).

Gold nanoparticle "bio-bar code" technology employs DNA oligomers to identify gold particles that are derivitized with antibodies to a specific target. Magnetic particles are also coated with antibodies to the same target, and are used to capture gold particles from the assay solution. The nanoparticle probe carries with it a large number of DNA oligomer probes, which are then released from the particle generating significant signal amplification when hybridized to a DNA array. Multiplexing is achieved through sequence specificity of oligomer hybridization to the array, and attomolar to zeptomolar $\left(10^{18}-10^{21}\right)$ sensitivity towards a target antigen has been reported using this method (Nam et al, 2002; Nam et al, 2003). These reagents are now commercially available from Nanosphere (www.nanosphere.com).

The combination of sensitive immunological detection with the parallel analysis of microarrays greatly enhances the ability of researchers to validate expression array data amongst protein subsets in a specific area of interest. Such techniques are only possible for specific endpoints, however, yet continuous dynamic analysis of multiple targets may soon be possible.

\section{QUANTUM DOTS AND REAL-TIME VISUALISATION}

Quantum dots (QDs) are promising nanostructures for a number of investigative biological applications. QDs are $2-8 \mathrm{~nm}$ semiconductor nanocrystals enclosed in a second semi-conductor shell with a larger spectral band-gap. When a QD absorbs a photon of a higher energy than the band-gap of the semiconductor from which it is constructed, a long-lifetime exciton is created, which decays with 5-40ns florescence emission (relative to $0.5-2 \mathrm{~ns}$ for conventional organic dyes). As 
such QDs are excellent candidates as fluorescent labels for detection of biomolecules by many types of microscopy. QDs have a narrow composition emission spectra, allowing for multiplexing of a number of QDs that are excited at the same wavelength, in theory generating a high capacity optical bar coding identification system.

QDs have been used to enhance the fluorescence of antibody detection in several assays; Wu et al report the use of a streptavidin coated QD conjugated that enhanced detection of a biotin tagged anti-Her2 antibody for an assay of SK-BR-3 breast cancer cells. QD-streptavidin conjugates have also been employed for intracellular detection of F-actin filaments in 3T3 fibroblasts, and quantum donor to fluorescent dye molecule acceptor ( $\mathrm{Cy} 5)$ interaction has been used to monitor in vitro hybridization of siRNAs to an QDlabeled mRNA target (Wu et al, 2003; Bakalova et al, 2005).

Dynamic visualization of proteins and nucleic acids within cells is limited by low intensity, short lifetimes and poor target accessibility of many conventional dyes. QDs offer enhanced sensitivity for detection of small number of macromolecules, and QDs have been shown capable of tracking internalized membrane protein receptors in cell culture, opening up the possibility for tracking vesicle associated cargos within cells in real-time. Rajan and $\mathrm{Vu}$ (2006) report the endocytosis of antibody conjugated QDs to follow internalization of the TrkA tyrosine kinase membrane receptor in a neuronal-like cell line. Endocytosis of QDs occurred during a 50 minute observation window and fluorescence was observed within the cells for up to 4 days, indicating the enhanced potential for QDs as biomarkers relative to conventional dyes. QDs were actively shuttled within neural processed in both retrograde and anteriograde directions, consistent with the immunohistochemical and GFP probes, indicating that QDs do not affect receptor trafficking. QD "blinking" - a property that identifies single of lownumber QD signals - suggested that single or small groups of receptors were being observed.

\section{SUMMARY}

Quantum dots and bio-bar coding technologies offer to greatly enhance the ability of researchers to monitor a wide range of targets at a sensitivity of just a few hundred molecules. Such highly sensitive detection and visualization will lead to a greater depth of understanding of spatial and temporal cellular events in a variety of models. These techniques are also amenable to adaptation and integration for "bio-chip" and nanofluidic devices - perhaps allowing analysis of multiple biological parameters from a single sample. It is to be hoped that design of computer software to integrate information will keep pace with the data generated by such inventive new analytical techniques.

\section{REFERENCES}

Bakalova R et al. 2005. Am Chem Soc, 127, 1132811335.

Mullenix M et al. 2004. In; Demidov VV and Broude NE (eds) DNA Amplification: Current Technologies and Applications, Boston University, USA, pp313-331, Nam JM et al. 2002. Science, 297, 1536-1540.

Nam JM et al. 2003. Science, 301, 1884-1886.

Rajan S et al. 2006. Nano Letts, 6, 2049-2059.

Sano T et al. 1992. Science, 230, 1350-1354.

Schweitzer B et al. 2000. Proc Nat Sci USA, 97, 1011310119.

Wu X et al. 2003. Nat Biotechnol, 21, 41-46. 